\title{
Improving the quality of transient response during automatic control of the turn of a tracked vehicle based on the implementation of structured input shapers
}

\author{
Igor Taratorkin ${ }^{1, *}$, Victor Derzhanskii ${ }^{1}$, and Alexander Taratorkin ${ }^{1}$ \\ ${ }^{1}$ IES UB RAS, Department of Transportation Vehicles Mechanics, 620049, Ekaterinburg, Russia
}

\begin{abstract}
The article presents the rationale for improving the quality of transients during steering control of a tracked vehicle in order to increase its high speed performance. Based on the comparative analysis of the results of simulation modeling and experimental research of the controlled movement dynamics, it is established that high speed performance is restricted by transient overshoot of a vehicle response to the control input, by transient oscillation and insufficient oscillation damping. The solution of the problem of high speed performance increase is carried out by implementing a modern IT approach - by creating an automated system for stabilizing the vehicle motion trajectory - which applies Input Shaper for adjusting the control input. The research has established that, considering the control object under research, the best results are obtained by Zero Vibration Shaper - (ZV-Shaper), which enables to minimize limiting overshoot, and the transient becomes close to an aperiodic one. The quality of the control process is defined by natural frequency stability of the system, to which the Input Shaper in question is tuned. In this regard, it is appropriate to introduce monitoring and identifying natural frequency into the control system. This creates conditions for increasing vehicle high speed performance, including on soils with bad traction properties.
\end{abstract}

One of the main operational properties of high-speed tracked vehicles is high speed performance represented by high-speed qualities. Increase in power-to-weight ratio in vehicles up to $25 \mathrm{~kW}$, improvement of transmissions, of motion control systems and data information systems provide for increase in potential high-speed qualities of modern tracked vehicles. However, the manifestation of potential high-speed qualities is limited by a number of dynamic phenomena, which characterize controllability at rectilinear motion and during turning, especially at vehicle robotization.

Severe vehicle operation conditions, limited possibilities for organizing maintenance service, insufficient reliability of electronic devices and hydrostatic drives, as well as cost limits for the steering control system lead to various suggestions on creating simple steering mechanisms and control systems. These systems have their own merits in terms of weight and volume indicators, simplicity, costs, a degree of design and technological perfection of the structural components. Steering devices with discrete properties, with which many pre-developed vehicles as well as the modern advanced TM-140 transport vehicle, intended for severe service conditions of the Arctic region, are equipped, can be referred to such control systems.

Moving along the roads with the hard surface coating (asphalt, concrete, frozen soil), with bad traction properties and intensive change of curvature, the high speed characteristics of the vehicles equipped with the steering control system (SCS) with discrete properties are limited and do not exceed $36 \ldots 38 \mathrm{~km} / \mathrm{h}$. It is explained by impossibility of variable (stepless) control of a trajectory curvature, angular acceleration, trajectory deviation compensation. At the same time, the intensity of the driver's control activity, i.e. the number of SCS actuations (steering control) per kilometer of driving increases by a factor of $6 . . .9$, reaching 96 . In this regard, the driver's compensatory control is effective at a speed lower than $36-38 \mathrm{~km} / \mathrm{h}$, and with application of remote control for robotic centers based on these vehicles, the average speed does not exceed $5 \mathrm{~km} / \mathrm{h}$. It determines the urgency of increasing high speed characteristics of tracked vehicles.

The goal (objective) of the research is to improve the quality of transients at controlling the angular speed of tracked vehicle cornering. The degree of implementing potential high-speed characteristics of the tracked vehicle is defined by the dynamic properties in case of coming into a corner (by a response to steering wheel jerk). For tracked vehicles with the steering mechanism providing for the discrete control of the driving direction, the angular speed $\omega$ undergoes discontinuity of the second kind, and its derivative $\dot{\omega}$ is the delta function $\delta(t)$, i.e. in absolute magnitude, it can change within an unlimited interval. However, the experimental data show that in an elastic-inertial mechanical system Track Assembly Transmission, which actuates friction elements slipping and interacts with soil by slipping, the value of the

\footnotetext{
* Corresponding author: ig tar@mail.ru
} 
derivative $\dot{\omega}$ changes continuously in terminal, though significant, limits. Even in case of a bounded deviation of angular speed up to $0.1 \mathrm{rad} / \mathrm{sec}$ with a frequency $2.5 \mathrm{~Hz}$ (due to drift and oscillatory processes in the powertrain), the angular accelerations reach $0.6 \mathrm{rad} / \mathrm{sec}^{2}$, and in case of cyclic actuation of the mechanism at long corners - up to $1.3 \mathrm{rad} / \mathrm{sec}^{2}$, and sideways accelerations - up to $10 \ldots 12$ $\mathrm{m} / \mathrm{s}^{2}$ [1]. In case of driving on hard surface soil, it is not possible to provide motion stabilization.

The specified features of the nonlinear control system with discrete properties are that at driving along the roads with an intensive change of curvature, the trajectory accuracy is reached by periodic steering brake actuation and its release, i.e. the control is pulsed. To provide a rationale for the ways of transient quality improvement, the track vehicle steering dynamics is simulated on the LMS Amesim platform [2]. The mathematical model of the plane-parallel controlled motion of the tracked vehicle as of a continuous solid body is described by the first two differential equations of the system (1). The third equation allows considering elastic-inertial properties of the dynamic parts of the system from the engine up to the driving sprockets of the vehicle.

$$
\begin{gathered}
m \ddot{y}+\frac{\dot{y}}{V} \sum K_{s_{i}}-\left[m V+\frac{1}{V} \sum K_{S_{i}} l_{i}\right] \dot{\varphi}_{t v}=0 \\
J_{z} \ddot{\varphi}_{t v}+\frac{\dot{\varphi}_{t v}}{V} \sum K_{S_{i}} l_{i}^{2}+\frac{y}{V} \sum K_{S_{i}} l_{i}+C_{t o r}\left[\varphi_{t v}-\varphi_{i c e}\right]=0 \\
J_{i c e} \ddot{\varphi}_{i c e}+C_{t o r}\left[\varphi_{i c e}-\varphi_{t v}\right]=M_{t u r n}
\end{gathered}
$$

where $y, \dot{y}, \ddot{y}, \varphi_{t v}, \dot{\varphi}_{t v}, \ddot{\varphi}_{t v} \varphi_{i c e}, \dot{\varphi}_{i c e}, \ddot{\varphi}_{i c e}$ are the generalized coordinates and their corresponding derivatives (at the lateral motion of the vehicle, yaw motion of the vehicle body in reference to the vertical axis and roll motion of the ICE crankshaft reduced to the angular coordinate of the vehicle body); $m$ - vehicle weight; $J_{z}$ - yaw moment of inertia; $J_{i c e}$ - the roll moment of inertia of the engine reduced to the angular coordinate of the vehicle body; $K_{s_{i}}$ - slipping resistance coefficient of the tracked vehicle; $C_{\text {tor }}$ - torsional rigidity of the transmission (main shaft) reduced to the angular coordinate of the vehicle body.

The value of the reduced rigidity $C_{t o r}$ can be considered as composition of the following components: rigidity of coupling shafts; driving track runs; suspension unit components of the lower rollers, as well as the stretching (tensioned) of the sagging upper tracks on the upper rollers [3]. This work considers the reduced rigidity as linearized. At track vehicle cornering, the values of the reduced rigidity and reduced moment of inertia of the engine depend on transmission ratio (the number of the engaged gear). The transients have been determined at a driving mode with a constant speed for a vehicle with the following parameters:

vehicle weight $m=11556 \mathrm{~kg}$; yaw moment of inertia $J_{z}=48200 \mathrm{~kg} \cdot \mathrm{m}^{2}$; slipping resistance coefficient $K_{s_{i}}=$ $60 \mathrm{kN} / \mathrm{rad}$; the sum of longitudinal coordinates of the lower roller axes in reference to the center of mass $\sum l_{i}=1.412 \mathrm{~m}$ and the sum of their squares $\sum\left(l_{i}\right)^{2}=7.06 \mathrm{~m}^{2}$; the reduced transmission rigidity $C_{\text {tor }}=84000 \mathrm{Nm} / \mathrm{rad}$. The reduced moment of inertia of the engine is determined considering the transmission ratio between the generalized coordinates of the engine (the third equation) and of the vehicle body in a rotary mode (the second equation), as well as the kinematic peculiarities of the vehicle steering mechanism. For the vehicle in question the reduced moment of inertia $J_{i c e}$ varies from $6148 \mathrm{~kg} \cdot \mathrm{m}^{2}$ up to $359589 \mathrm{~kg} \cdot \mathrm{m}^{2}$. The value of the yaw moment was set in the form of the step function imitating the vehicle going into a corner, at the same time, the maximum value was taken to be equal 0.9 from the greatest possible under the motion conditions, which is determined by cornering resistance coefficient.

The simulation results show that the parameters of the transient function significantly depend on a driving speed. The transient is accompanied by oscillations starting with the third gear $(5.6 \mathrm{~m} / \mathrm{sec})$. The intensity of damping is determined by the soil damping properties. The response overshoot at the top gear reaches $50 \%$, which demands speed restriction according to safety conditions.

The obtained results suggest that the discrete properties of the steering control system restrict high speed characteristics of vehicles because of oscillation transients and significant response overshoot in case of going into a corner. In engineering, such phenomena are described as a Bonanza effect $[4,5]$, which is observed in the course of controlling transport vehicle mechanical systems. In general, generated oscillations restrict high speed characteristics of vehicles, they are perceived by the driver as disturbing ones, increase structural components load and decrease the comfort level. The suggested measures are necessary to reduce oscillation amplitude by means of frequency detuning, i.e. the maximum amplitudes will be shifted at frequencies which are not critical for the vehicle. The kinetic energy of an oscillatory process depends on moment $\mathrm{M}$ squared and is inversely proportional to the doubled value of the reduced rigidity $\mathrm{C}$.

Rigidity increase leads to decrease in oscillation kinetic energy because of the torque impulse created by the steering control system. It results in higher efficiency of damping with all other things being equal, but is, as a rule, infeasible for design, configuration or technological reasons. At invariable design parameters of the vehicle, as an alternative, the impulse which causes oscillations at torque application can be changed to prevent or reduce generation of natural oscillations. The procedure can be explained by means of a practical example described by R Fischer [6].

It appears that the most effective way of restricting oscillations is introduction into the steering and brake control unit of Input Shapers. The main idea of these shapers consists in formation of the controlling signal by convolution of the controlling input with the pulse sequence in the form of the Dirac delta function. Shaper operating algorithm is based on redistribution in time of power impact on a controlled object preserving an invariable value of the total impact. Due to relative increase in time of a system transient from one state into another, compensation of the arising oscillations is provided, and the shift of the control (response, impact) for a fraction of the period of the calculated oscillations of the controlled object provides ideally their full suppression. At the same time, the reference-input signal 
is accepted such as the resultant effect on the system remained invariable:

$$
\sum_{i=1}^{n} A_{i}=1
$$

where $\mathrm{n}$ is the number of pulses; $A_{i}$ is the amplitude of the $i^{\text {th }}$ pulse.

The key parameter defining the quality of shapingalgorithm performance is the level of oscillation suppression. It is worthwhile making an estimation of suppression in the form of the ratio of the residual oscillation amplitude in the system in which the input signal was created by the shaping-filter to the amplitude of residual oscillations which would arise in the same system without conversion of an input signal. The degree of oscillation suppression is defined by the following expressions [7]:

$$
\left[\begin{array}{c}
V(\omega, \varepsilon)=e^{-\varepsilon \omega t_{n}} \sqrt{C^{2}+D^{2}} \\
C(\omega, \varepsilon)=\sum_{i=1}^{n} A_{i} e^{-\varepsilon \omega t_{i}} \cos \left(\omega t_{i} \sqrt{1-\varepsilon^{2}}\right) \\
C(\omega, \varepsilon)=\sum_{i=1}^{n} A_{i} e^{-\varepsilon \omega t_{i}} \sin \left(\omega t_{i} \sqrt{1-\varepsilon^{2}}\right)
\end{array}\right.
$$

where $\omega$ is an oscillation frequency; $\varepsilon$ is a damping coefficient; $t_{i}$ is time of the $i^{\text {th }}$ pulse.

Shaping-filter parameters of the $A_{i}$ and $t_{i}$ are received by solving the equations (2) and (3), being defined by the maximum allowable level of oscillations $V(\omega, \varepsilon) \max$.. At the same time, the values $\omega$ and $\varepsilon$ are usually selected equal to the natural frequency and to the controlled object damping coefficient, which can be determined by a mathematical model or by the results of the experimental findings. It should be considered that real-life controlled objects (in particular the steering control system of the tracked vehicle) are non-linear that leads to changes of the specified parameters. Thus, there appears a task of shaping such an input signal which will provide robustness in the system in relation to uncertainty of generated oscillation frequency (natural frequency) and to the controlled object damping coefficient. One of the solutions of this task is introduction of an additional condition:

$$
\frac{\partial}{\partial \omega}\left(e^{-\varepsilon \omega t_{n}} \sqrt{C^{2}+D^{2}}\right)=0
$$

The partial derivative of the expression (4) considering frequency being equal to zero allows for increasing a range of frequencies, in which the desirable degree of oscillation suppression is provided, and by that achieving acceptable Input Shaping performance even in case of a significant error in determination of controlled object natural frequency[7].

Another important property characterizing the quality of Input Shapers performance is high-speed characteristics which is defined by the time of the latest pulse $t n$. For this reason, when calculating the algorithm, a condition of $t n$ minimization is usually entered. In such a case, it is necessary to introduce restrictions for pulse minimization amplitude as far as time minimization can result in the necessity of shaping pulses with an infinite amplitude. Execution of the given conditions is defined by the dynamics of the object under research and by the choice of a Shaper-algorithm type.
Shaping-algorithms have found a wide circulation in different industrial applications [8] that allowed for increasing the performance of different devices considerably: of cranes $[8,9,10]$, coordinate measuring machines [11,12], satellite systems [13], milling machines [14], disk drives [15] and others. The above-mentioned references describe the types of Shapers, dependences on their parameter determination, efficiency assessment for different industrial applications and recommendations about their use.

The key parameter according to which an algorithm type is selected is a natural frequency of the system. For the object under study, the frequency value is defined by the number of engaged gear. In this regard, a possibility of applying one algorithm of tracked vehicle steering control for all the gears is assessed. Fig. 1. shows the assessment of oscillation suppression efficiency in case of application of different Shaping-algorithms. The obtained data show that in the range of natural frequencies $1,4 \ldots$ $3,92 \mathrm{rad} / \mathrm{s}$ (in case of tuning the Input Shaper for the frequency corresponding to the $5^{\text {th }}$ gear - direct-driven gear) a satisfactory result is achieved in case of control system tuning according to 3-EI -Shaper-algorithm. In this case, the level of residual oscillations in the range of frequencies under study does not exceed 5\% from the amplitude of the residual oscillations which arise in the same system without input signal conversion.

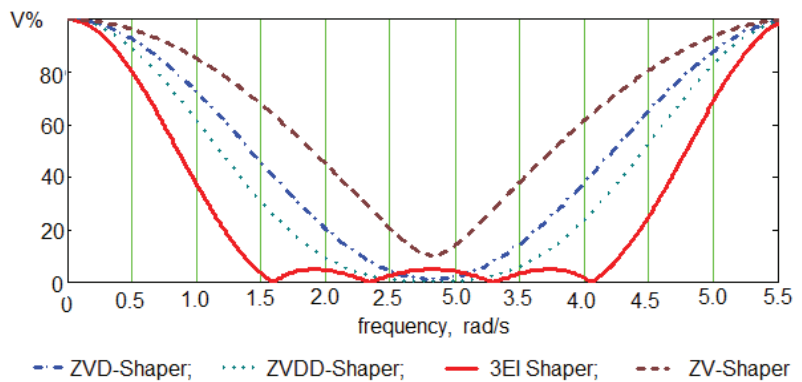

Fig. 1. Assessment of oscillation suppression efficiency by different Input Shaping (residual oscillation)

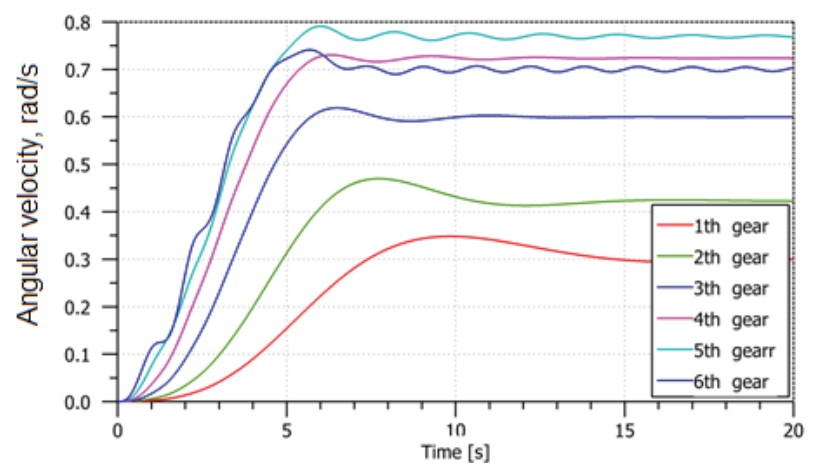

Fig. 2. Assessment of high-speed performance of the system with 3-EI - Input Shaping

However, in this case the required high-speed performance of the vehicle steering control system is not provided as far as the transient duration time increases fourfold (see Fig. 2.) and reaches 5 seconds. In this respect, the use of the simplest ZV, ZVD - Shapers provides for satisfactory high-speed performance (the 
obtained level of the angular speed is reached for $1.5 \ldots$ $2.0 \mathrm{sec}$ in comparison with $1.1 \mathrm{sec}$ for the option without Shaping application) in case of tuning up for one frequency. Furthermore, the required robustness level is not provided (see Fig. 3.). Fig. 3. shows that in case of the effective oscillation damping at the fifth gear (ZV-Shaper is tuned up for the frequency of $0.45 \mathrm{~Hz}$ and corresponds to natural frequency at the fifth, "direct-driven" gear), the degree of oscillation suppression at other gears is not satisfactory. The similar results are provided by the Shapers working according to a negative algorithm - UMZV Shapers, etc.

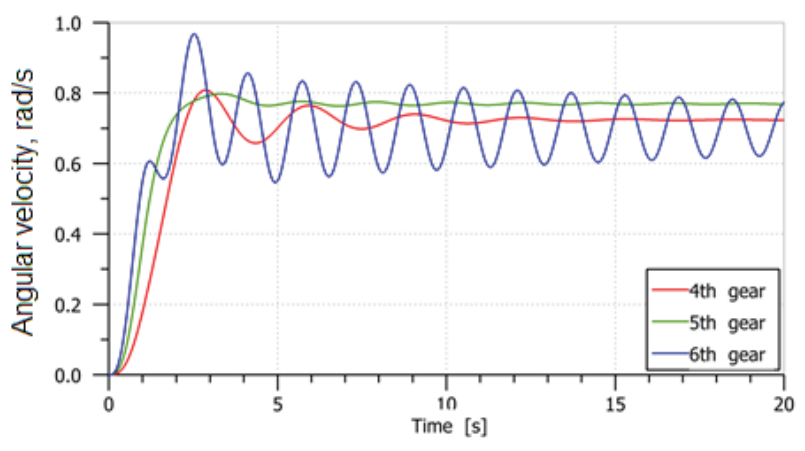

Fig. 3. Assessment of ZV-Shaper Quality on high-speed performance and robustness

In this case, the application of negative algorithms is restricted by the speed change of the controlling (force) input. These algorithms with the specified negative amplitude (SNA) in case of the value of the of the amplitude ratio of two adjacent pulses in the range from 0.5 to 2 allows for reaching a compromise between the requirement of increasing algorithm high-speed performance and reducing the power of the steering control drive. In relation to the research process of tracked vehicle steering, the restriction is caused by impossibility of quick creation of a braking effort at the speeding up vehicle side, owing to the necessity of changing the driving and loose tracks in the track chain. Consideration of this circumstance does not allow for using these algorithms in relation to the research object in the steering control process.

On the basis of the study undertaken, it may be concluded that input Shapers can be applied in the steering control system of the tracked vehicle. In the system under study, the change of system natural frequency at the specified gears in the gearbox can have significant effect on the parameters of a transient. It can be caused both by nonlinearity of the system and by change of the vehicle weight in case of different rates of loading capacity, fuel load, tyre temperature of the road wheels leading to a considerable change of the slipping resistance coefficient, etc.

Comparing different input Shapers, it can be concluded that the best effect considering high-speed performance is reached either by application of ZV and ZVD algorithms, or by algorithms with pulses of different signs UM - ZV, however, the property of robustness decreases. As the key property of the steering control system of the tracked vehicle is high-speed performance, so ZV - Shaper application with introduction into the control system of monitoring and natural frequency identification is preferable. Application of this Input Shaping allows for minimizing the ceiling overshoot up to $5 \%$, and the transient becomes close to aperiodic, including driving with high speed on soils with bad traction properties.

\section{Restrictions}

The research is executed on the basis of analyzing the linear model of the dynamic system. The reduced rigidity $C_{\text {tor }}$ in the real-life system is significantly nonlinear and asymmetric, which can excite parametrical oscillations [16]. Besides, there is a good reason to consider the longitudinal displacement of the vehicle turning center and nonlinearity of lateral slipping resistance coefficient $K_{s_{i}}$. Taking into account the abovementioned, repeatability of the results of the experimental study and simulation, can be increased.

\section{Conclusion}

As a result of simulation and experimental study of the dynamics of the controlled movement of the tracked vehicle with the discrete properties of the steering control system, it is established that the extent of realization of potential high-speed qualities is limited to the quality of response to a steering wheel jerk (to a single control input).

To achieve a required transient control quality at tracked vehicle cornering, there is a good reason to apply input Shapers in the control system providing optimum ratio among contradictory properties of high speed performance, an extent of oscillation suppression and robustness.

At driving on the roads with bad traction properties, high-speed qualities can be increased by introduction into the turning brake control unit of a ZV Shaper providing prevention of oscillation generation at response overshoot which does not exceed $5 \%$.

\section{References}

1. A. Taratorkin, V. Derzhanskii, I. Taratorkin, Pr. Eng., 136, 1368-1377 (2016) - $\quad$ DOI 10.1016/j.proeng.2016.07.331

2. LMS Imagine.Lab Model-oriented approach to designing mechatronic systems. URL: https://www.plm.automation.siemens.com/ru $\mathrm{ru} /$ products/lms/imagine-lab/amesim

3. V. Derzhanskii, I. Taratorkin, SAE Technical Papers, 9, (2013)

4. G. Jurgens, R. Fischer, 697, VDI-Berichte, Dusseldorf, 233, (1988)

5. H-J Schspf, G Jurgens, R Fischer, 91, ATZ, 568-575, (1989)

6. R. Fischer, F. Küçükay, G. Jürgens, R. Najork, B. Pollak, DOI 10.1007/978-3-319-05263-2, (2015)

7. N.C. Singer, W.P. Seering, 112, 76-82, (1990) 
8. K.L. Sorensen, A Thesis Presented to The Academic Faculty, ( 2008)

9. K.L. Sorensen, W.E, Singhose, S. Dickerson, 15, № 7, 825-837, (2007)

10. A.P. Kuznetsov, A.V. Markov, A.S. Shmarlouski, T.V. Gavrilik, Doklady BGUIR, 6(60), 5-12, (2011)

11. N. Seth, K. Rattan, R. Brandstetter // IEEE Conf. On Control Apps., 368-373, (1993)

12. S. Jones, A.G. Ulsoy // J. of Dynamic Systems, Measurement, and Control, 121, № June. 242-247, (1999)

13. W.E. Singhose, L.J. Porter, T.D. Tuttle et al. // J. of Dynamic Systems, Measurement, and Control, 119, № June, 320-326, (1997)

14. J. Fortgang, W. Singhose, J. Marquez et al., // Proceedings of theAmerican Control Conf., 45314536, (2005)

15. W. Singhose, W.P. Seering, N.C. Singer, J. of Dynamic Systems, Measurement and Control, 119, № June, 198-205, (1997)

16. A. Taratorkin, V. Derzhanskii, I. Taratorkin, LNEE, 10, 495-504, (2013) 